\title{
Efectos de una cuota compensatoria ad valorem sobre importaciones de carne de pollo en México
}

\author{
Effects of an ad valorem compensatory quota on chicken meat imports in Mexico \\ Pedro Hernández-Aguirre, Samuel Rebollar-Rebollar2², Germán Gómez-Tenorio², \\ Héctor Hugo Velázquez-Villalva²
}
${ }^{1}$ Posgrado en Ciencias Agropecuarias y Recursos Naturales, Centro Universitario UAEM, Universidad Autónoma del Estado de México (UAEM), Carr. Toluca-Tejupilco Km. 67.5, Barrio de Santiago, 51300, Temascaltepec, Estado de México, México. ${ }^{2}$ Centro Universitario UAEM Temascaltepec, UAEM.

*Autor para correspondencia: srebollarr@uaemex.mx

\section{Fecha de recepción:}

17 de enero de 2020

Fecha de aceptación:

4 de junio de 2020

Disponible en línea:

29 de julio de 2020

Este es un artículo en acceso

abierto que se distribuye de

acuerdo a los términos de la

licencia Creative Commons.

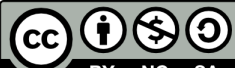

Reconocimiento-

NoComercial-CompartirIgual

4.0 Internacional

\section{RESUMEN}

Se evaluó el efecto regional de una cuota compensatoria ad valorem de $25.7 \%$ en importaciones de carne de pollo en México, con datos de 2018. Para ello, México se dividió en ocho regiones productoras, ocho consumidoras y dos puntos de internación del producto. Se utilizó un modelo de programación no lineal y su visualización sobre producción, importaciones, consumo y en el Valor Social Neto (VSN). Con el modelo base, la producción, las importaciones y el consumo fueron de 3,338,824; 770,050; y 4,108,872 t de carne de pollo y un VSN de 2,491 miles de millones de pesos; con la cuota compensatoria de $25.7 \%$, la producción aumentó $0.3 \%$, el consumo se redujo $1.4 \%$, los precios al productor y al consumidor aumentaron $0.3 \%$ y el VSN descendió $0.3 \%$. Se concluye que una cuota compensatoria benefició a productores, pero perjudicó a consumidores nacionales, al pagar un precio mayor, y ocasionó una reducción en el bienestar de la sociedad.

PALABRAS ClAVE

Carne de pollo, tarifa, programación no lineal, Valor Social Neto.

\section{ABSTRACT}

We evaluated the regional effect of an ad valorem countervailing duty of $25.7 \%$ on chicken meat imports into Mexico with data from 2018. Mexico was divided into eight producing regions, eight consumers areas and two points of product entry. We used a non-linear programming model and its visualization on production, imports, consumption and net social value (NSV). With the base model, production, imports and consumption amounted to 3,338,824; 770,050; and $4,108,872 \mathrm{t}$ of poultry meat, and an NSV of 2,491 billion pesos; with the compensatory quota of $25.7 \%$, production increased by $0.3 \%$, consumption decreased by $1.4 \%$, producer and consumer prices increased by $0.3 \%$ and the NSV decreased by $0.3 \%$. The conclusion was that producers were benefited by the compensatory quota, national consumers were affected by having to pay a higher price, and the welfare of society was reduced.

KEYWORDS

Poultry meat, tariff, non-linear programming, Net Social Value. 


\section{INTRODUCCIÓN}

La industria y las actividades productivas desempeñan un papel importante para la economía y el desarrollo de un país, por lo que se vuelve indispensable generar o mantener altos niveles de competitividad y de demanda. Adicionalmente, los gobiernos deben responsabilizarse de mantener a salvo sus industrias de aquellas prácticas que puedan afectar la competencia justa, sobre todo cuando el intercambio comercial con economías fuertemente exportadoras es considerable (Kerr y Loppacher 2004). Por tal razón, se establecen medidas específicas o encaminadas hacia la consecucion de este objetivo, como las cuotas compensatorias (Núñez et al. 1999), las cuales se encargan de equilibrar condiciones de competencia entre productores nacionales y exportadores que importan mercancías mediante prácticas desleales de comercio internacional (Kerr y Loppacher 2004; Cruz-Barney 2007). Éste es el caso de la rama de la producción nacional (Saldaña-Pérez 2011) referente a la carne de pollo (pierna y muslo) en México. Las prácticas desleales se refieren a conductas mercantiles que se presentan en intercambios de bienes al aprovechar la apertura de mercados y desregulaciones comerciales en general (Núñez et al. 1999; Witker y Hernández 2005; Cruz-Barney 2007).

En México, la única medida de salvaguardia definitiva que se ha impuesto en contra de las importaciones de pollo originarias de Estados Unidos de América (EUA) es de carácter bilateral (DOF 2002); sin embargo, el 17 de septiembre de 2018, la Secretaría de Economía de México (SE) publicó en el Diario Oficial de la Federación (DOF) la resolución final del examen de vigencia de la cuota compensatoria impuesta a importaciones de pierna y muslo de pollo (definidos, técnicamente, como la parte del cuerpo del ave - en este caso, del pollo- que comprende la pierna, el muslo y su unión, excluyendo patas, alas, pechuga y cabeza) (DOF 2018), originarios de Estados Unidos, provenientes de Simmons, Sanderson, Tyson y Pilgrim's Pride, a través de las fracciones arancelarias 0207.13.03 y 0207.14.04 de la TIGGIE (Tarifa de la Ley de los Impuestos Generales de Importación y Exportación) o de cualquier otra (DOF 2018). En dicha resolución, se dio a conocer la prórroga de la vigencia de la cuota compensatoria por cinco años más, contados a partir del 7 de agosto de
2017, y se asume, de acuerdo con lo estipulado en esa resolución, que en 2022 deberá terminar esa vigencia (SE 2018).

Al respecto, cifras oficiales señalan que México no es autosuficiente en consumo nacional de carne de pollo; pues, mientras que en 1990, el país consumió 1.7 millones de toneladas (Mt), de las cuales, 1.2 millones se produjeron al interior y la diferencia se compró, principalmente de EUA y de Brasil, en 2018, la cifra se incrementó en promedio anual $5.4 \%$, para ubicarse en 2.3 Mt (1.9 millones fueron producción interna, y la diferencia, importaciones). En todo ese periodo, la producción aumentó 3.4\% y las compras externas $4.7 \%$ (SIAP 2019).

Si bien, debido al TLCAN (Tratado de Libre Comercio de América del Norte), México importa carne de pollo, sobre todo pierna y muslo desde EUA y Brasil con arancel de cero a partir de 2004 (Saldaña-Pérez 2011), desde 2018, productores mexicanos de carne de pollo ejercen presión sobre el Gobierno Federal para que establezca e imponga una cuota compensatoria a importaciones provenientes de EUA, con el argumento de que esa carne entra a México a precios por debajo del costo de producción doméstico, lo cual ha dañado la producción nacional (DOF 2018). Los productores nacionales han presentado al Gobierno (Secretaría de Economía) evidencia de investigaciones previas sobre importaciones a precios dumping, con el propósito de que se apruebe la imposición de una cuota compensatoria equivalente a $25.7 \%$ (DOF 2018) y, con ello, para el mexicano sea indiferente consumir carne de pollo producida en México o carne importada.

Desde la publicación de la resolución final, las circunstancias esenciales que dieron lugar a la decisión de la SE de imponer cuotas compensatorias no se han modificado. Continuaron las ventas por debajo de los costos de producción de la pierna y el muslo de pollo en EUA, así como las prácticas de discriminación de precios de los avicultores estadounidenses; por ello, al no aplicarse las cuotas compensatorias, las crecientes importaciones mexicanas de dicha carne - producto de los bajos precios de exportación y los grandes excedentes de pierna y mulso en el mercado estadounidense - continuaron causando daño a la rama de la producción nacional de este subproducto pecuario (Saldaña-Pérez 2011; DOF 2018). Por lo anterior, el objetivo de esta investigación consistió en determinar 
el efecto de la aplicación de una cuota compensatoria de $25.7 \%$ a importaciones de carne de pollo que lleva a cabo México y sus efectos sobre la producción, importaciones, consumo y precios al productor y al consumidor, tanto por región como en el total nacional, así como en el bienestar de la sociedad, medido por el Valor Social Neto (VSN). La hipótesis principal señala que una cuota compensatoria incrementa la producción de carne de pollo de forma regional y nacional; reduce el consumo y las importaciones de esa carne; incrementa los precios al productor y al consumidor de carne de pollo, y reduce el bienestar de la sociedad, perjudicando a consumidores nacionales de carne de esa ave.

\section{Materiales y métodos}

Para evaluar el efecto de la cuota compensatoria de 25.7\% a importaciones mexicanas de carne de pollo, se utilizó un modelo de programación no lineal con funciones inversas de demanda y oferta de carne de pollo (también conocidas como funciones de precios endógenos) (Rebollar-Rebollar et al. 2019c), con datos de 2018. Con base en Bassols (1992), México se dividió en ocho regiones productoras, ocho regiones consumidoras y dos puntos de internación de importaciones de carne de pollo. Las regiones y sus entidades fueron: Noroeste (NO): Baja California, Baja California Sur, Sonora, Sinaloa y Nayarit; Norte (NR): Chihuahua, Coahuila, Durango, Zacatecas y San Luis Potosí; Noreste (NE): Nuevo León y Tamaulipas; Centro-Occidente (CO): Colima, Jalisco, Aguascalientes, Michoacán y Guanajuato; Centro-Este (CE): Querétaro, Hidalgo, Tlaxcala, Puebla, Morelos, Ciudad de México y Estado de México; Sur (SU): Guerrero, Oaxaca y Chiapas; Oriente (OR): Tabasco y Veracruz; Península de Yucatán (PE): Campeche, Yucatán y Quintana Roo.

Referente a los puntos de internación de las importaciones de carne de pollo, el primero de ellos (PI1) se conformó por las aduanas de Colombia, perteneciente al estado de Nuevo León; Nuevo Laredo, Matamoros y Reynosa, en el estado de Tamaulipas, y Piedras Negras, en el estado de Coahuila. Este primer punto registró el ingreso de $68 \%$ de la carne importada. El punto de internación dos (PI2) integró las aduanas de Mexicali, Ensenada y Tijuana, ubicadas en Baja California; Nogales y San Luis Río Colorado, como parte del estado de Sonora, y Ciudad Juárez, perteneciente al estado de Chihuahua. Por este punto ingresó 32\% de la importación de carne de pollo (SIAP 2019). Se decidió utilizar la regionalización del país, debido a que el consumo de carne de esta especie pecuaria no es homogéneo en todo el país; éste se lleva a cabo de forma regionalizada (Huerta-Sanabria et al. 2018), y, entre regiones, el consumo no es igual; por tanto, el efecto de variables económicas que lo determinan es distinto en cada una de ellas.

\section{Las ecuaciones}

La ecuación de demanda-precio, funcionalmente dependiente de la cantidad demandada (función inversa) (Vázquez-Alvarado y Martínez-Damián 2011;

$$
P_{d i}=P_{d i}\left(Y_{d i}\right)=\lambda_{d i}+\omega_{d i} Y_{d i} ; \omega<0
$$

Rebollar-Rebollar et al. 2019b), para la región $i$ fue:

Donde:

$\mathrm{P}_{\mathrm{di}}=$ precio de demanda de carne de pollo en canal en la región $i$, en pesos por tonelada.

$\lambda_{\mathrm{di}}=$ intercepto de la función de demanda de carne de pollo en canal para la región $i$.

$\omega_{\mathrm{di}}=$ pendiente de la función de demanda de carne de pollo en canal para la región $i$.

$\mathrm{Y}_{\mathrm{di}}=$ cantidad demandada de carne de pollo en canal en la región $i$, en toneladas.

Para la misma región, la función inversa del modelo de

$$
P_{s i}=P_{s i}\left(X_{s i}\right)=\mathrm{V}_{s i}+\eta_{s i} X_{s i} ; \beta>0
$$

oferta es la siguiente:

Donde:

$P_{s i}=$ precio de oferta de carne de pollo en canal en la región $i$, en pesos por tonelada.

$v_{s i}=$ intercepto de la función de oferta de carne de pollo en canal en la región $i$.

$\eta_{s i}=$ pendiente de la función de oferta de carne de pollo en canal en la región $i$.

$X_{s i}=$ cantidad ofrecida de carne de pollo en canal en la región $i$, en toneladas.

Con lo anterior, la función de cuasi bienestar social para cada región (1) se definió por el área entre la curva de demanda de carne de pollo y el área entre 
la curva de oferta, dadas por $Y_{d i}^{*}$ y $X_{s i^{\prime}}^{*}$ las cuales representan las cantidades óptimas de carne de pollo que es

$$
W_{i}\left(Q_{s i}^{*}, Q_{d i}^{*}\right)=\int_{0}^{Y_{d i}^{*}} P_{d i}\left(Y_{d i}\right) d Y_{d i}-\int_{0}^{X_{s i}^{*}} P_{s i}\left(X_{s i}\right) d X_{s i}(1)
$$

necesario obtener:

Cuando se incorporan los costos de transporte entre regiones, la función de bienestar social (función objetivo) para

$$
\operatorname{Max} \sum_{i=1}^{n}\left[\int_{0}^{Y_{d i}^{*}} P_{d i}\left(Y_{d i}\right) d Y_{d i}-\int_{0}^{X_{s i}^{*}} P_{s i}\left(X_{s i}\right) d X_{s i}\right]-\sum_{i=1}^{n} \sum_{j=1}^{n} X_{i j} T_{i j}
$$

las $n$ regiones (2) se representa de la siguiente manera:

Donde:

$X_{i j}=$ cantidad transportada de carne de pollo de la región $i$ a la región $j(\$ / t)$ de carne de pollo en canal.

$T_{i j}=$ costo de transporte de la región $i$ a la región $j(\$ / t)$ de carne de pollo en canal.

Componentes adicionales del modelo fueron las restricciones de demanda y de oferta. Las de demanda (3) implican

$$
Y_{d i} \leq \sum_{j=1}^{n} T_{i j} \text {, para toda } i
$$

que la suma de la cantidad transportada a la región $i$ debe ser mayor o igual que la demanda de esa región. Esto es: Las restricciones de oferta (4) implican que la suma de la cantidad transportada fuera de la región $i$ debe ser menor

$$
X_{s i} \geq \sum_{j=1}^{n} T_{i j}, \text { para toda } i(4)
$$

o igual a la producción total de carne de pollo de esa región:

Takayama y Judge (1964) y Rebollar-Rebollar et al. (2019b) afirman que en el modelo se asume la existencia de regiones productoras y consumidoras que comercian un bien homogéneo; en este caso, ese bien homogéneo es la carne de pollo en canal. Tales regiones se hallan separadas por los costos de transporte, pero no aisladas entre sí.

$$
\operatorname{Max} V S N=\sum_{i=1}^{8}\left[\lambda_{d} Y_{d}+\frac{1}{2} \omega d Y_{d}^{2}\right]-\sum_{s=1}^{10}\left[V_{s} X_{s}+\frac{1}{2} \eta_{s} X_{s}^{2}\right]-\sum_{s=1}^{8}\left[t_{s d} X_{s d}\right]-\sum_{s=1}^{2} t_{s d}+\text { cuota }
$$

En su representación matemática, el resultado de la integral de [1] es el modelo matemático como sigue:

La palabra cuota en esta última ecuación significa que la cuota compensatoria ad valorem se agregó a los costos de transporte relacionados con los puntos de internación o con las importaciones de carne de pollo en canal.

\section{Datos}

La información sobre consumo (demanda) de cada una de las ocho regiones del país provino de utilizar el dato de la población de cada estado durante 2018, registrada por el Consejo Nacional de Población (CONAPO 2018). Después, el dato de población se multiplicó por el consumo per cápita de carne de pollo de 2018, reportado por la Unión de Nacional de Avicultores (UNA) (UNA 2018) y se sumó el consumo de cada uno de los estados que integran cada región para generar el total regional referente a dicho consumo. Por el lado de la oferta, el precio de carne de pollo regional se obtuvo mediante el precio de cada entidad federativa que integró la región, ponderado con la producción de esa misma región; la información provino del Sistema de Información Agroalimentaria y Pesquera (SIAP). El precio de la carne de pollo proveniente de Estados Unidos hacia los dos puntos de internación se obtuvo del Sistema Nacional de Información e Integración de Mercados (SNIIM 2018).

Por su parte, el dato referente al costo de transporte dentro y entre las regiones se calculó al multiplicar la distancia (en kilómetros), por el costo por kilómetro; las distancias se obtuvieron con la herramienta de la Secretaría de Comunicaciones y Trasportes (SCT 2019); se consideraron las capitales de las entidades federativas como punto de referencia y se ponderaron las distancias en cada región. En México, la carne de pollo se transporta al movilizar el ave in vivo; no se 
trasladan canales frías ni canales calientes; por ello, este transporte es especial, en el sentido de que se utilizan cajas específicas donde se colocan las aves. El costo por kilómetro fue de 44 pesos mexicanos en el momento de la investigación, y se obtuvo al consultar los índices de precios en el transporte por carretera de la Secretaría de Comunicaciones y Transporte; asimismo, se consiguió el dato correspondiente al costo del diésel en la composición porcentual del gasto, para lo cual se consideró la inflación del mismo año. Para estimar las funciones inversas de demanda y oferta de carne de pollo para cada región de México, se utilizó la elasticidad precio de la demanda y elasticidad precio de la oferta regional estimadas por Rebollar-Rebollar et al. (2019), y para los puntos de internación se consideraron las elasticidades estimadas por Vázquez-Alvarado y Martínez-Damián (2011). Las funciones inversas de demanda y oferta de carne de pollo se estimaron de acuerdo con Alston et al. (1995) y Kawaguchi et al. (1997). Dispuesta toda la información oficial sobre carne de pollo en canal, elasticidades, ecuaciones de demanda y oferta, precios al productor, al consumidor, etc., se procedió a estimar el modelo de programación no lineal, conocido como modelo base o modelo óptimo, y los resultados, tanto por región como nacional, sobre producción, importaciones, consumo y en el VSN.

El modelo base u óptimo es aquel estimado sin distorsiones de mercado o aquel al que no se le ha aplicado el análisis de algún tipo de política o escenario (Rebollar-Rebollar et al. 2019b).

Una diferencia porcentual de estimación de la producción, importaciones y consumo entre el modelo base y los datos observados en el año de análisis menor a $10 \%$ significa que el modelo estimado es aceptable para llevar a cabo el análisis de escenarios de política. Si tal diferencia en el resultado de la estimación es negativa/ positiva, se dice que el modelo ha subestimado o sobreestimado los resultados (Rebollar-Rebollar et al. 2019c). El porcentaje de la cuota compensatoria ad valorem de $25.7 \%$ (DOF 2018) se aplicó en la matriz del costo por tonelada transportada de las importaciones o sobre el costo de transporte del producto de los dos puntos de internación de la carne de pollo hacia cada una de las regiones del país. Como ejemplo, en el esquema del modelo base, el costo de la carne de pollo importada que se consideró fue de 44.3 pesos, el cual se sumó al costo de transporte de los puntos de internación uno y dos, y, al agregarle $25.7 \%$ como cuota compensatoria, representó un aumento de 11.4 pesos por kilogramo, y un nuevo precio de 55.7 pesos por kilogramo, que se suman (se le agregaron 11.4 pesos por kilogramo) al costo de transporte de la carne de pollo importada de los dos puntos de internación.

Asimismo, el modelo base consideró un costo de transporte del PI1 a la región NO, en 2018, de 6,949 pesos mexicanos por tonelada; por tanto, con el escenario, ahora el dato que se consideró fue $\$ 6,949$ $+\$ 8,892=\$ 15,841$ por tonelada, y así sucesivamente para las regiones Norte, Noreste, Centro-Occidente, Centro-Este, Sur, Oriente y Península. El análisis de resultados del modelo de programación no lineal con el escenario de la cuota compensatoria de $25.7 \%$ se hizo sobre producción, importaciones, consumo, precios al productor, precios al consumidor y VSN; éstos se contrastaron con los del modelo base (modelo óptimo), con el objetivo de visualizar e interpretar los efectos del escenario (cuota compensatoria) sobre el mercado de la carne de pollo en México.

Toda la información se procesó con el software Solver MINOS, escrito en el lenguaje de programación GAMS (General Algebraic Modeling System), versión 24.4.2, para Windows, Office 2013.

\section{Resultados Y Discusión}

Respecto a la información oficial de México, observada durante 2018, el modelo de programación no lineal estimado - conocido como modelo base o modelo óptimo (Rebollar-Rebollar et al. 2019b) - sobreestimó la producción mexicana de carne de pollo en $0.01 \%$; esto es, la maximización del VSN mostró que la producción nacional del modelo óptimo $(3,338,824)$ se ubicó en $451 \mathrm{t}$ de carne de pollo superior al volumen observado $(3,338,373 \mathrm{t})$ de dicha carne en ese año (Cuadro 1$)$.

La sobreestimación en el volumen de producción de ese subproducto pecuario se presentó solamente en tres de las ocho regiones de México, con predominancia en la Centro-Este (CE) (3.2\%). Adicionalmente, el modelo subestimó la producción de esa carne en la Península de Yucatán (PE) (-6\%), en la Norte (NR) $(-1.4 \%)$, Centro-Occidente $(\mathrm{CO})(-1 \%)$, Oriente $(\mathrm{OR})$ $(-0.03 \%)$ y Noroeste $(\mathrm{NO})(-0.04 \%)$, cuyos volúmenes reportados fueron menores a los que se observaron 
(Cuadro 1). La expresión subestimó significa que el resultado del modelo base o modelo óptimo fue menor al dato observado en el año de análisis. En relación con los puntos de internación, el modelo óptimo subestimó el total de importaciones que entraron por el punto de internación uno (PI1) y por el punto de internación dos (PI2) en 3,656 (0.7\%) y 3,021 t (1.2\%); esto es, para maximizar el VSN, la cantidad de carne de pollo que ingresó por esos dos puntos fue menor a lo que se observó en ese año, es decir, una subestimación total equivalente a $0.9 \%$.

En cuanto al consumo, el modelo base subestimó el total óptimo nacional al observar una diferencia de 8,470 t $(0.2 \%)$ menos entre el valor estimado por el modelo y el observado en el año de análisis; sin embargo, en cuatro de las ocho regiones, hubo sobreestimación en el consumo, y, en términos relativos, destacaron la NO (1.4\%), CO (0.2\%), PE $(0.1 \%)$ y la NR $(0.03 \%)$ (Cuadro 1). El VSN óptimo del mercado de carne de pollo en México (el del modelo base) fue mayor al observado en $10 \%$; esto significa que, a nivel nacional, el modelo sobreestimó producción nacional y subestimó tanto las importaciones como el consumo, aunque no fue así a nivel región (Cuadro 1).

Para maximizar el VSN, la estimación del modelo de programación (modelo óptimo) sugirió que el abasto y la distribución óptima de carne de pollo en México, entre regiones, debió efectuarse de acuerdo con lo que se observa en el Cuadro 2. En las filas se ubicaron el volumen de producción y los envíos óptimos de carne de pollo de cada región, y, en las columnas, lo que cada región consumió de esa carne, de tal forma que la suma total de las filas fue igual a la suma total de las columnas.

Cuadro 1. México. Carne de pollo en canal. Modelo base u óptimo. 2018.

\begin{tabular}{|c|c|c|c|c|}
\hline Región & Observado & Modelo base & Cambio & Cambio \% \\
\hline \multicolumn{5}{|c|}{ Producción $(\mathrm{t})$} \\
\hline Noroeste (NO) & 203,313 & 203,241 & -72 & -0.04 \\
\hline Norte (NR) & 471,390 & 465,039 & $-6,351$ & -1.37 \\
\hline Noreste (NE) & 68,843 & 70,701 & 1,858 & 2.63 \\
\hline Centro-Occidente (CO) & $1,044,478$ & $1,034,013$ & $-10,465$ & -1.01 \\
\hline Centro-Este (CE) & 757,180 & 782,018 & 24,838 & 3.18 \\
\hline Sur (SU) & 210,117 & 210,530 & 413 & 0.20 \\
\hline Oriente (OR) & 413,467 & 413,347 & -120 & -0.03 \\
\hline Península de Yucatán (PE) & 169,585 & 159,935 & $-9,650$ & -6.03 \\
\hline \multicolumn{5}{|c|}{ Importaciones $(\mathrm{t})$} \\
\hline Punto de internación 1 & 528,174 & 524,518 & $-3,656$ & -0.70 \\
\hline Punto de internación 2 & 248,553 & 245,532 & $-3,021$ & -1.23 \\
\hline \multicolumn{5}{|c|}{ Consumo (t) } \\
\hline Noroeste (NO) & 391,710 & 397,236 & 5,526 & 1.39 \\
\hline Norte (NR) & 433,290 & 433,424 & 134 & 0.03 \\
\hline Noreste (NE) & 295,680 & 295,233 & -447 & -0.15 \\
\hline Centro-Occidente (CO) & 691,020 & 692,101 & 1,081 & 0.16 \\
\hline Centro-Este (CE) & $1,357,290$ & $1,349,942$ & $-7,348$ & -0.54 \\
\hline Sur (SU) & 433,950 & 430,814 & $-3,136$ & -0.73 \\
\hline Oriente (OR) & 352,440 & 350,187 & $-2,253$ & -0.64 \\
\hline Península de Yucatán (PE) & 159,720 & 159,935 & 215 & 0.13 \\
\hline VSN (MMDP) & 2,242 & 2,491 & 249 & 10.00 \\
\hline
\end{tabular}

Fuente: elaboración propia con resultados del modelo base. MMDP: Miles de millones de pesos. Nota: en este cuadro se utilizaron dos decimales en la última columna para valorar la diferencia del cambio. 
El modelo óptimo maximizó el VSN, en contraste con el observado en el año de análisis; así, el VSN óptimo fue superior al observado en $10 \%$, equivalente a 249 billones de pesos. El volumen producido de carne de pollo nacional otorgado por el modelo fue superior al observado en $0.01 \%$ (Cuadro 1 ); el incremento se dio sólo en tres de las ocho regiones de México (CE, NE y $\mathrm{SU})$, con predominancia en la NE. Aquéllas en las que se observó un descenso mayor en la producción de carne de pollo fueron la PE, NR y CO.

El total de importaciones de esta carne asignadas por el modelo base decreció en $0.9 \%(6,677$ t), en relación con el volumen observado durante el año de análisis. El modelo reasignó un volumen menor de carne de pollo por el PI1, equivalente a 3,656 (0.7\%), y de 3,021 t (1.2\%), por el PI2, que no ingresó al país por esas aduanas, debido tanto a las distancias como a costos de transporte del producto hacia los centros de consumo de México (Cuadro 1). De forma similar, la maximización del VSN redujo el consumo óptimo nacional de carne de pollo en $0.2 \%$ (8,470 t); esa disminución se observó en las regiones $\mathrm{CE}, \mathrm{SU}, \mathrm{OR}$ y NE (quienes recibieron $100 \%$ de las importaciones de carne de pollo que ingresaron por el PI1); en tanto que en NO, CO, PE y la NR, el consumo de este producto aumentó. Todas las importaciones de carne de pollo que ingresaron por el PI2 se consumieron solamente en las regiones NE y NR (Cuadro 2).

En relación con el abasto y consumo de carne de pollo (Cuadro 2), el modelo base indicó que la región
NO consumió 401,859 t de carne de pollo; de ese total, consumió todo lo que produjo y recibió producto (49.4\%), por medio de importaciones que entraron por el PI2. La región CE fue deficitaria en producción de carne de pollo, pero fue la que más consumió esa carne (33.9\% del total nacional); sin embargo, para solventar su consumo interno $(1,407,303$ t), la maximización del VSN indicó que esta región tuvo que enviar 20.1\% de su producción hacia la región SU de México y, a su vez, recibió carne de pollo de las regiones CO (24.3\%), NR $(5.9 \%)$ y NE (5\%) e importaciones que ingresaron por el PI1 (20.4\% de su consumo total) (Cuadro 2). Por su parte, de acuerdo con el modelo base, la región de la PE fue autosuficiente en consumo de carne de pollo, pues todo lo que produjo se consumió en la misma región, no recibió producto de ninguna otra región de México y tampoco hubo importaciones.

\section{Análisis del efecto de la cuota compensatoria ad valorem}

Como se mencionó, una cuota compensatoria se establece cuando el país que importa demuestra evidencia de que la mercancía ingresó a precios dumping o mediante subvenciones (Núñez et al. 1999; Witker y Hernández 2005). Al respecto, México, nunca ha hecho efectiva una cuota compensatoria a importaciones de pierna y muslo de pollo procedentes de los Estados Unidos, Brasil o de cualquier otro país (SR 2019); equivocadamente, impuso una medida de

Cuadro 2. Matriz de abasto y distribución de carne de pollo en México, 2018, cifras en toneladas.

\begin{tabular}{|c|c|c|c|c|c|c|c|c|c|}
\hline Región & $\mathrm{NO}$ & NR & $\mathrm{NE}$ & $\mathrm{CO}$ & $\mathrm{CE}$ & SU & OR & $\mathrm{PE}$ & Total \\
\hline $\mathrm{NO}$ & 200,406 & & & & & & & & 200,406 \\
\hline NR & & 433,475 & & & 33,901 & & & & 467,376 \\
\hline NE & & & & & 71,076 & & & & 71,076 \\
\hline $\mathrm{CO}$ & & & & 687,126 & 342,144 & & & & $1,029,270$ \\
\hline CE & & & & & 614,054 & 172,549 & & & 786,603 \\
\hline SU & & & & & & 210,032 & & & 210,032 \\
\hline OR & & & & & & 49,125 & 349,495 & & 398,620 \\
\hline PE & & & & & & & & 159,942 & 159,942 \\
\hline PI1 & & & 295,173 & & 287,047 & & & & 582,220 \\
\hline PI2 & 198,623 & 27,655 & & & & & & & 226,273 \\
\hline Total & 399,029 & 461,130 & 295,173 & 687,126 & $1,348,222$ & 431,706 & 349,495 & 159,942 & $4,131,823$ \\
\hline
\end{tabular}

Fuente: elaboración propia, con resultados del modelo base. 
salvaguardia bilateral a las importaciones de pollo estadounidense, para tratar de resolver un problema que en el fondo fue de discriminación de precios o de dumping (Saldaña-Pérez 2011); por ello, sólo ha publicado resoluciones de exámenes de vigencia (DOF 2002, 2018) en los que determinó no aplicarlas para no sobredimensionar su efecto en el mercado (principalmente en los precios al consumidor), en tanto no se regularicen situaciones derivadas de la contingencia sobre el virus de la Influencia Aviar tipo A, subtipo H7N3, en algunos estados de la nación norteamericana (TIGGIE 2007; DOF 2018).

Con base en las condiciones en las que se planteó esta investigación, si México en algún momento decidiera hacer efectiva la cuota compensatoria de
$25.7 \%$, los efectos esperados en el mercado de la carne de pollo son los que se describen a continuación. Con una cuota compensatoria de $25.7 \%$, se estimó que la producción de carne de pollo, tanto nacional como por regiones, aumentaría $0.3 \%$, equivalente a $8,446 \mathrm{t}$ de carne (Cuadro 3).

Sin embargo, las regiones de México que recibieron el mayor impacto debido a la cuota compensatoria fueron la Centro-Este (CE) y la Centro-Occidente (CO), mismas que han tenido más dinamismo en esta actividad pecuaria en México (Rebollar-Rebollar et al. 2019a; Rebollar-Rebollar et al. 2019b). En relación con el modelo base, la cuota compensatoria de $25.7 \%$ redujo el volumen nacional de importaciones de carne de pollo en $1.4 \%$, equivalente a 10,755 t; de las cuales,

Cuadro 3. Cuota compensatoria de $25.7 \%$ y sus efectos sobre el mercado de la carne de pollo en México, 2018.

\begin{tabular}{|c|c|c|c|c|}
\hline Región & Modelo base & $\begin{array}{c}\text { Cuota } \\
\text { compensatoria } \\
25.7 \%\end{array}$ & Cambio (t) & Cambio \% \\
\hline \multicolumn{5}{|c|}{ Producción (t) } \\
\hline Noroeste (NO) & 203,241 & 203,242 & 1 & 0.000 \\
\hline Norte (NR) & 465,039 & 465,487 & 448 & 0.001 \\
\hline Noreste (NE) & 70,701 & 71,584 & 883 & 0.012 \\
\hline Centro-Occidente (CO) & $1,034,013$ & $1,036,849$ & 2,836 & 0.003 \\
\hline Centro-Este (CE) & 782,018 & 786,010 & 3,992 & 0.005 \\
\hline Sur (SU) & 210,530 & 210,596 & 66 & 0.000 \\
\hline Oriente (OR) & 413,347 & 413,567 & 220 & 0.001 \\
\hline Península de Yucatán (PE) & 159,935 & 159,935 & 0 & 0.000 \\
\hline \multicolumn{5}{|c|}{ Importaciones (t) } \\
\hline Punto de internación 1 & 524,518 & 517,204 & $-7,314$ & -0.014 \\
\hline Punto de internación 2 & 245,532 & 242,091 & $-3,441$ & -0.014 \\
\hline \multicolumn{5}{|c|}{ Consumo (t) } \\
\hline Noroeste (NO) & 397,236 & 396,965 & -271 & -0.001 \\
\hline Norte (NR) & 433,424 & 433,114 & -310 & -0.001 \\
\hline Noreste (NE) & 295,233 & 295,124 & -109 & 0.000 \\
\hline Centro-Occidente (CO) & 692,101 & 691,471 & -630 & -0.001 \\
\hline Centro-Este (CE) & $1,349,942$ & $1,349,394$ & -548 & 0.000 \\
\hline Sur (SU) & 430,814 & 430,667 & -147 & 0.000 \\
\hline Oriente (OR) & 350,187 & 349,895 & -292 & -0.001 \\
\hline Península de Yucatán (PE) & 159,935 & 159,935 & 0 & 0.000 \\
\hline VSN (MMDP) & 2,491 & 2,484 & -7 & -0.003 \\
\hline
\end{tabular}

Fuente: elaboración propia, con resultados del modelo base y de la cuota compensatoria. Nota: se escribieron hasta tres décimos con el propósito de observar el cambio en porcentajes. 
7,314 t (68\%) dejaron de ingresar por el PI1 y el resto (32\%) por el PI2 (Cuadro 3); este resultado es similar al de Trueta-Santiago y Heberth-Hernández (2004), enfocado a que una cuota compensatoria sobre importaciones mexicanas de carne bovina procedentes de EUA, reducen el volumen de las compras externas. Además, con la cuota compensatoria de $25.7 \%$, el consumo total nacional se afectó con una reducción de 0.004\% (177 t).

En términos absolutos, el impacto de la cuota compensatoria de $25.7 \%$ fue más notorio en las regiones mexicanas del Centro-Occidente (CO) y en la Centro-Este (CE), equivalente a una reducción en el consumo de 630 y 548 t, al considerar que el precio recibido por el productor con el efecto de la cuota compensatoria en esas regiones fue de $\$ 31,881$ y $\$ 32,941$ por tonelada, en relación con los del modelo base. Así, la pérdida de valor por este subproducto cárnico que se dejó de enviar hacia esas zonas fue cercano a los 40 millones de pesos; en tanto que, en la región de la Península (PE), el impacto de la cuota compensatoria fue nulo, debido a que esa región es autosuficiente en el mercado de la carne de pollo.

Con la cuota compensatoria de $25.7 \%$ sobre importaciones de carne de pollo procedentes de EUA, el modelo generó un VSN menor al que se obtuvo con el modelo óptimo, afirmación similar al estudio que efectuaron Núñez et al. (1999) sobre importaciones mexicanas de dos productos chinos. Al aplicarse la medida (la cuota compensatoria), el bienestar de la sociedad - medido por el VSN - fue menor en $0.3 \%$, equivalente a 6.8 mil millones de pesos (Cuadro 3); el resultado concuerda con los hallazgos de Aaron y Pierce (2019) sobre pérdida de bienestar por efectos de cuotas de represalia impuestas por EUA a productos manufactureros chinos. Con referencia al Cuadro 4 , con la cuota compensatoria de $25.7 \%$ a la carne de pollo en México, tanto los precios al consumidor como al productor se incrementaron, excepto en la región de la Península de Yucatán.

Los precios al productor fueron mayores debido al efecto de la cuota compensatoria sobre la producción nacional y en la producción por región de esta carne; esta afirmación es similar al hallazgo de Villa-Hernández et al. (2019), gracias al cual concluyeron que la aplicación de aranceles a productos agropecuarios provenientes de EUA generó incrementos de precios en los tres sectores económicos de México, y convergente con Aaron y Pierce (2019), quienes han observado que los precios al productor de EUA aumentaron por efecto de este tipo de cuotas.

Con base en los resultados del modelo base, la cuota compensatoria de $25.7 \%$ redujo las importaciones y el consumo de carne de pollo en México, $y$, en consecuencia, su efecto se observó en un aumento de los precios que pagó el consumidor, por una aparente escasez relativa de esta carne en el mercado. Concordante con estos resultados, Amiti et al. (2019) concluyeron que la incidencia total de las políticas comerciales proteccionistas de EUA durante 2018, cayó sobre consumidores, importadores nacionales y provocó reducción en el ingreso disponible de ese país. A eso se refirió el Gobierno de México cuando decidió no hacer efectiva la aplicación de la cuota compensatoria de $25.7 \%$ a las importaciones de carne de pollo (TIGGIE 2007; DOF 2018).

Asimismo, esta cuota disminuyó los precios de la carne que ingresó por los puntos de internación, resultado similar al efecto esperado por la imposición de una cuota compensatoria de $17.5 \%$ sobre el consumidor estadounidense de tomate mexicano (Asci et al. 2016; USDC 2019; SE 2019). Así, con la cuota compensatoria de $25.7 \%$, la variable precio al productor del PI1 experimentó una reducción de $29.6 \%$ (\$8,784 por t), al pasar de $\$ 29,638$ por tonelada sin la cuota compensatoria a \$20,854 con el efecto de la cuota, situación similar con el precio al productor referente al PI2 (Cuadro 4).

\section{CONCLUSIONES}

Con base en los resultados, con la aplicación de la cuota compensatoria de $25.7 \%$ a importaciones de carne de pollo provenientes de Estados Unidos, los productores fueron los beneficiados por el incremento en el volumen de producción nacional y por el incremento de los precios recibidos. En los puntos de internación, la cuota compensatoria redujo los precios al productor, pero perjudicó a consumidores nacionales porque enfrentaron un incremento en los precios pagados por esa carne; lo anterior se tradujo en una pérdida del bienestar de la sociedad, porque, con la cuota compensatoria, el VSN se redujo. Finalmente, el análisis de la cuota compensatoria a importaciones de carne de pollo brinda elementos 
Cuadro 4. Efectos de la cuota compensatoria en los precios al productor y al consumidor de carne de pollo en México.

\begin{tabular}{lrrrr}
\hline \multicolumn{5}{c}{ Precios óptimos $(\$ / \mathrm{t})$} \\
\hline Región & Consumidoro base & Productor & Consumidor & Productor \\
\hline NO & 30,510 & 27,013 & 30,620 & 27,121 \\
NR & 31,950 & 30,599 & 32,060 & 30,707 \\
NE & 30,990 & 30,759 & 31,090 & 30,867 \\
CO & 32,500 & 31,773 & 32,610 & 31,881 \\
CE & 33,410 & 32,833 & 33,520 & 32,941 \\
SU & 34,650 & 32,916 & 34,750 & 33,024 \\
OR & 33,590 & 32,664 & 33,700 & 32,772 \\
PE & 34,460 & 33,865 & 34,460 & 33,865 \\
PI1 & \multicolumn{5}{c}{} & 20,854 \\
PI2 & 29,638 & 17,750 \\
\hline
\end{tabular}

Fuente: elaboración propia con resultados del modelo base 2018 y del modelo con la cuota compensatoria en carne de pollo.

que México tendría que considerar si decidiera hacer efectiva la aplicación de dicha cuota.

\section{AGRADECIMIENTOS}

Este trabajo fue producto del proyecto de investigación "Comportamiento de la oferta y demanda regionales de carne de pollo en México", con clave de registro 4217/2016/SF", autorizado por la Universidad Autónoma del Estado de México. 


\section{LITERATURA CITADA}

Aaron F, Pierce J. 2019. Disentangling the effects of the 20182019 tariffs on a globally connected U.S. manufacturing sector. Finance and economics discussion series 2019086. Board of Governors of the Federal Reserve System Washington, USA.

Alston JM, Norton W, Prdey G. 1995. Science under Scarcity: Principles and Practice for Agricultural Research Evaluation and Priority Setting. Cornell University Press. Ithaca, USA.

Amiti M, Redding SJ, Weinstein DE. 2019. The Impact of the 2018 trade war on U.S. price and welfare. Journal of Economic Perspectives 33(4): 187-210. https://doi. org/10.1257/jep.33.4.187

Asci S, Seal J, Onel G, VanSickle J. 2016. US and Mexican tomatoes: Perceptions and implications of the renegotiated suspensión agreement. Journal of Agricultural and Resource Economics 42(1): 138-160.

Avicultura. [internet]. 2018. ¿Se hará efectiva la aplicación de cuotas compensatorias por dumping del pollo? [cited 2019 Nov 3]. Disponible en: https:// www.avicultura.mx/destacado/\%C2\%BFSe-hara-efectiva-la-aplicacion-de-las-cuotas-compensatorias-por-dumping-del-pollo.

Bassols BA. 1992. México: formación de regiones económicas. Primera reimpresión. Instituto de Investigaciones Económicas. UNAM. México, D. F.

[CONAPO] Consejo Nacional de Población. [internet]. 2018. Proyecciones de la población de los municipios de México, 2015-2030. [cited 2019 Feb 20]. Disponible en: https://www.gob.mx/conapo/documentos/proyecciones-de-la-poblacion-de-los-municipios-de-mexico-2015-2030.

Cruz-Barney O. 2007. Antecedentes del sistema contra prácticas desleales de comercio en México. La evolución de las disposiciones antidumping. Boletín Mexicano de Derecho Comparado 119: 439-465.

[DOF] Diario Oficial de la Federación. [internet]. 2002. Resolución por la que se acepta la solicitud de parte interesada y se declara el inicio de la investigación de medidas de salvaguarda bilateral sobre las importaciones de piernas y muslos de pollo, originarias de los Estados Unidos de América. [cited 2019 Oct 12]. Disponible en: http://dof. gob.mx/index.php?year=2002\&month=11\&day=22.

[DOF] Diario Oficial de la Federación. [internet]. 2018. Resolución final del examen de vigencia de la cuo- ta compensatoria impuesta a las importaciones de pierna y muslo de pollo originarias de los Estados Unidos de América, independientemente del país de procedencia. [cited 2019 Oct 13]. Disponible en: http://www.dof.gob.mx/nota_detalle. php? codigo $=5535960 \&$ fecha $=27 / 08 / 2018$.

Huerta-Sanabria S, Arana-Coronado OA, SagarnagaVillegas LM, Matus-Gardea JA, Brambila-Paz JJ. 2018. Impacto del ingreso y carencias sociales sobre el consumo de carne en México. Revista Mexicana de Ciencias Agrícolas 9(6): 1245-1258.

Kawaguchi T, Susuki N, Kaiser M. 1997. A Spatial equilibrium model for imperfectly competitive milk markets. American Journal of Agricultural Economics 79: 851-859. https://doi.org/10.2307/1244426

Kerr AW, Loppacher JL. 2004. Anti-dumping in the Doha negotiations-Fairy Tales at the WTO. Journal of World Trade 38(2): 211-244.

Núñez G, Rodríguez M del R, de la Cruz S. 1999. Cálculo del impacto de una cuota compensatoria en el bienestar de los consumidores: un caso ilustrativo. Estudios Económicos 11(1): 141-160.

Rebollar-Rebollar E, Gómez-Tenorio G, RebollarRebollar A, Mondragón-Ancelmo J. 2019. Oferta y demanda regional de carne de pollo en México. Revista Mexicana de Ciencias Pecuarias 10(4): 917-932.

Rebollar-Rebollar E, Hernández-Martínez J, RebollarRebollar S, Gómez-Tenorio G, Rebollar-Rebollar A. 2019a. Dinámica y especialización regional de la producción de carne de pollo en México, 2004-2017. Investigación y Ciencia 78: 14-22.

Rebollar-Rebollar E, Rebollar-Rebollar S, GuzmánSoria E, Gómez-Tenorio G. 2019b. Efecto de las importaciones en la oferta regional de carne de pollo en México, 1996-2016. Tropical and Subtropical Agroecosystems 22: 415-425.

Rebollar-Rebollar S, Chiatchoua C, Gómez-Tenorio G. 2019c. Efectos de la aplicación de un impuesto en México: caso carne de cerdo. Análisis Económico 34(86): 245-261.

Saldaña-Pérez JM. 2011. Importaciones de pollo de los Estados Unidos. Un caso de dumping, no de salvaguardia. Cultura Jurídica 1: 15-41.

[SCT] Secretaría de Comunicaciones y Transportes. [internet]. 2019. Rutas punto a punto. [cited 2019 Feb 3]. Disponible en: http://app.sct.gob.mx/sibuac_internet/ ControllerUI?action $=\mathrm{cmdSolRutas}$. 
[SE] Secretaría de Economía. [internet]. 2018. Mercancías sujetas a cuotas compensatorias. [cited 2019 Oct 12]. Disponible en: https://www.derecho.unam.mx/ investigacion/publicaciones/revista-cultura/pdf/ CJ(Art_1).pdf.

[SE] Secretaría de Economía. [internet]. 2019. Acuerdo de suspensión del tomate mexicano en Estados Unidos. [cited 2020 Jun 5]. Disponible en: https://www.gob. $\mathrm{mx} / \mathrm{se} /$ prensa/comunicado-de-prensa-sobre-el-acuerdo-de-suspension-del-tomate-mexicano-en-estados-unidos?idiom=es.

[SR] Senado de la República. [internet]. 2019. Cupo para importar con el arancel-cupo establecido carne de pollo. [cited 2020 May 17]. Disponible en: https://infosen. senado.gob.mx/sgsp/gaceta/64/2/2019-11-14-1/assets/ documentos/PA_CAGPDR_Arancel_Cupo.pdf.

[SIAP] Servicio de Información Agroalimentaria y Pesquera. [internet]. 2019. Avance de la producción pecuaria por estado. [cited 2019 Feb 10]. Disponible en: http://infosiap.siap.gob.mx/repoAvance_siap_gb/ pecAvanceEdo.jsp.

[SNIIM] Sistema Nacional de Información e Integración de Mercados. [internet]. 2018. Mercados nacionales pecuarios: Carne de ave. [cited 2019 Ene 20]. Disponible en: http://www.economia-sniim.gob.mx/nuevo/ Home.aspx?opcion=/SNIIM-Pecuarios-Nacionales $/$ MenAve.asp.

Takayama T, Judge G. 1964. Spatial equilibrium and quadratic programming. Journal of Farm Economics 46(1): 67-93. https://doi.org/10.2307/1236473

[TIGGIE] Tarifa de la Ley de los Impuestos Generales de Importación y Exportación. [internet]. 2007. Tarifa 2007 vigente. [cited 2019 Oct 25]. Disponible en: http:// www.siicex-caaarem.org.mx/Bases/TIGIE2007.nsf/ 4caa80bd19d9258006256b050078593c/5a3dd5f689a3cb aa862573020072a1ab?OpenDocument.

Trueta-Santiago R, Heberth-Hernández J. 2004. Los costos del aperturismo a ultranza: Consecuencias comparativas para los productores mexicanos y norteamericanos. Revista Mexicana de Agronegocios 8(14): 221-235.

[UNA] Unión Nacional de Avicultores. [internet]. 2018. Indicadores económicos. [cited 2019Feb 20]. Disponible en: https://una.org.mx/indicadores-economicos/.

[USDC] US Department of Commerce. [internet]. 2019. U.S. Department of Commerce Announces a New Draft Suspension Agreement on Fresh Tomatoes from Mexico I U.S. Department of Commerce. [cited 2020
Jun 5]. Disponible en: https://www.commerce.gov/ news/press-releases/2019/08/us-department-commerce-announces-new-draft-suspension-agreement-fresh.

Vázquez-Alvarado JMP, Martínez-Damián MA. [internet]. 2011. Elasticidades de oferta y demanda de los principales productos agropecuarios de México. Instituto Nacional de Investigaciones Forestales Agrícolas y Pecuarias (INIFAP). [cited 2019 Oct 28]. Disponible en: http://siafemor.inifap.gob.mx/anec/ pdf/descargables/54/54.pdf.

Villa-Hernández YA, Kido-Cruz A, Hernández-Silva V, Madrigal-Moreno S. 2019. Efecto de la imposición de aranceles a productos agropecuarios de Estados Unidos en importaciones de México, TLCAN. Paradigma Económico 1(11): 163-182.

Witker J, Hernández S. 2005. Resolución de controversias en materia de antidumping y cuotas compensatorias en el TLCAN. Revista Latinoamericana de Derecho 2(3): 273-314. 